\title{
Classification of transient power quality disturbance based on S-transform and fuzzy KNN
}

\author{
Hong Men ${ }^{1}$ a , Jia Liu ${ }^{1}$, Yilong Hao ${ }^{1}$, Chunlai $\mathrm{Li}^{2, \mathrm{~b}}$ \\ ${ }^{1}$ School of Automation Engineering, Northeast Dianli University, Jilin China 132012 \\ ${ }^{2}$ State Grid Qinghai Electric Power Research Institute, Xining China 810008 \\ aemail: menhong_bme@163.com, b415064774@qq.com
}

Keywords: Transient power quality disturbance; Intelligent classification; S-transform; Fuzzy KNN

\begin{abstract}
This paper proposed a new method to classify transient power quality disturbances using S-transform and K-Nearest Neighbor classifier. First, S-transform was applied to perform time-frequency analysis on the power quality disturbance signals, and the S-transform mode time-frequency matrix was achieved from the S-transform, and we got 8 valid features extracted from the matrix, then the feature vectors who consisted of the former features were input into the Fuzzy KNN classifier to perform the disturbing signals intelligent classification, then completed the intelligent classification for six types of transient power quality disturbances signals. The result showed that the structure of the proposed method was simple, the number of the needed features was little and it was doing well in classifying these types of transient power quality disturbances.
\end{abstract}

\section{Introduction}

The transient power quality disturbance is electric power operation problem that electric power departments and users have given special attention in recent years. Disturbance problems would cause serious consequences such as the un-normal run of the sensitive load, damage of the power electronic devices, restart of the microprocessor intelligent devices and failure of protection ${ }^{[1-2]}$. Therefore it is necessary to take identification and classification of the various kinds of transient power quality disturbance signals for the control of the power quality.

As the inheritance and development of the wavelet transform and Fourier transform, the S-transform uses the Gaussian window function to dispose the signals. The inverse of the width and frequency has a directly proportional relationship and the result is intuitive and not easy to be affected by noises ${ }^{[3-5]}$. The result of S-transform is a plural time-frequency matrix which contains distribution information such as amplitude, frequency and phase; the matrix is suitable for the signal feature extraction of the transient power quality disturbance signals ${ }^{[6-8]}$. $\mathrm{KNN}(\mathrm{K}-\mathrm{Nearest}$ Neighbor) algorithm is a type of mature algorithm in theory and has advantages as simple structure, fast training speed etc ${ }^{9]}$. Fuzzy k nearest neighbor algorithm(Fuzzy KNN) is an improved algorithm that combines the fuzzy theory and KNN. In this paper, Matlab was used to simulate 6 kinds of common transient power quality disturbance signals, and feature extraction algorithm was proposed based on the S-transform, combined with the fuzzy KNN classifier, the transient power quality disturbance signal was classified automatically. The flow block diagram of the classification of the transient power quality disturbance signal based on S-transform is shown in figure 1.

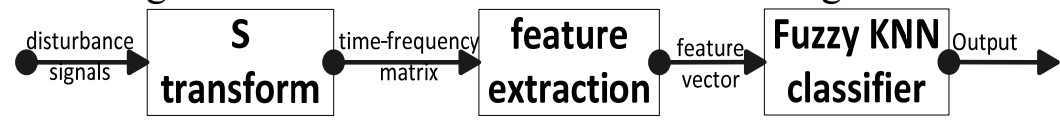

Fig.1 Flow block diagram of the classification of the transient power quality disturbance signal based on S-transform

In this paper, part 1 described the basic principle of S-transform briefly, part 2 described the detection and analysis of the transient power quality disturbance signal based on S-transform, part 3 described the feature extraction part, part 4 described Fuzzy KNN, part 5 described the analysis of the results of simulation, part 6 described the conclusion. 


\section{Principle of S-transform}

S-transform was a kind of reversible time-frequency analysis method which was first proposed in 1996by scholar Stock well etc. It was a development of continuous wavelet transform and short-time Fourier transform ${ }^{[10]}$.

The one-dimensional continuous S-transform of signal $\mathrm{h}(\mathrm{t})$ was defined as follow:

$$
\begin{aligned}
& S(f, \tau)=\int_{-\infty}^{\infty} x(t) \frac{|f|}{\sqrt{2 \pi}} e^{\frac{(\tau-t)^{2} f^{2}}{2}} e^{-j 2 \pi f t} d t \\
& w(t)=\frac{1}{\sigma \sqrt{2 \pi}} e^{\frac{t^{2}}{2 \sigma^{2}}}
\end{aligned}
$$

Among which, $\omega(t)$ is Gaussian window function, $\tau$ is the shift factor, $f$ is frequency, $j$ is the imaginary unit.

Set $\delta=1 /|\mathrm{f}|, \delta$ was the standard variance of the corresponding Gaussian function to control the width of the window function, so that the width of the Gaussian window would change with the change of the frequency. When in the low frequency interval, the frequency resolution was high as the Gaussian window was wide and flat; When in the high frequency interval, the frequency resolution was high as the Gaussian window was tall and high. This kind of multi-resolution time-frequency analysis method met the needs of the transient signal analysis and overcome the defects that the time-frequency resolution of short-time Fourier transform was changeless.

The one-dimensional continuous inverse transformation:

$$
x(t)=\int_{-\infty}^{\infty}[S(f, \tau)] e^{j 2 \pi f t} d f
$$

The relationship between S-transform of signal $\mathrm{x}(\mathrm{t})$ and Fourier transform $\mathrm{x}(\mathrm{f})$ :

$$
X(f)=\int_{-\infty}^{\infty} S(f, \tau) d \tau
$$

Therefore, discrete S-transform could be gained through the following ways: Assume h(kT)(k=0, $1,2, \ldots, \mathrm{N}-1)$ was the discrete time series sampled from the continuous time signal $\mathrm{x}(\mathrm{t})$, the sampling interval was $\mathrm{t}$, the total sampling points was $\mathrm{N}$.

The discrete Fourier transform:

$$
\begin{aligned}
& S\left[\frac{n}{N T}, k T\right]=\sum_{m=0}^{N-1} X\left[\frac{m+n}{N T}\right] e^{-\frac{2 \pi^{2} m^{2}}{n^{2}}} e^{\frac{j 2 \pi m k}{N}} \\
& (k, m=0, \cdots 1, \cdots, N-1 ; \cdots n=1, \cdots, N-1)
\end{aligned}
$$

S-transform could be calculated rapidly by fast Fourier transform, in particular, when $\mathrm{n}=0$, the discrete S-transform:

$$
S[0, k T]=\frac{1}{N} \sum_{m=0}^{N-1} x\left(\frac{m}{N T}\right)
$$

A complex time-frequency matrix was got through S-transform from the signal $\mathrm{x}(\mathrm{kT})$, denoted as $\mathrm{S}$ matrix, the column elements corresponded to the time sampling points, the line elements corresponded to the frequency sampling points. Molded the elements of S matrix to get the mode $\mathrm{S}$ time-frequency matrix, the column vector represented the distribution the signal amplitude varied with the frequency transformation at a certain moment, the row vector represented the distribution the signal amplitude varied with the frequency transformation at a certain moment. So the size of any element in the $\mathrm{S}$ mode time-frequency matrix was the corresponding amplitude size of the frequency and time in S-transform ${ }^{[11]}$.

$\mathrm{N}^{*} \mathrm{~N}$ points ( $\mathrm{N}$ rows and $\mathrm{N}$ columns) would be got by calculation steps from $\mathrm{S}$-transformation of $\mathrm{N}$ signal. But the $\mathrm{S}$-transform mode time-frequency matrix had $\mathrm{N} / 2+1$ rows and $\mathrm{N}$ columns, as the S-transform needed $\mathrm{N} / 2+1$ times $\mathrm{N}$ points fast Fourier transform for the conjugate symmetry characteristic of the Fourier transform. 


\section{Detection and analysis of the transient power quality disturbance signal based on S-transform}

Six types of signals was produced using the MATLAB simulation, based on the mathematical model of the disturbance signals in literature ${ }^{[12]}: \mathrm{S} 1$ - the ideal voltage signal, $\mathrm{S} 2$ - the transient decline voltage signal, S3 - the transient rise voltage signal, S4 - the interrupt voltage signal, S5 the transient oscillation signal, S6 - the transient harmonic signal. Among which the power frequency is $50 \mathrm{~Hz}$. The sampling frequency of the high frequency oscillation signal was $3200 \mathrm{~Hz}$ while the sampling frequency of the rest was $1000 \mathrm{~Hz}$. The amplitude parameters, the disturbance moment start-stop parameters, disturbance duration and frequency parameters of the disturbance signal were generated randomly for a better simulation of the actual situation. The mode time-frequency matrix was obtained by the s-transform, and the corresponding time domain and frequency domain characteristic curve were obtained by line vector envelope curve and column vector envelope curve respectively. Figure a, b, c, d, e, f represented the ideal voltage signal, the transient decline voltage signal, the transient rise voltage signal, the interrupt voltage signal, the transient oscillation signal, and the transient harmonic signal respectively.

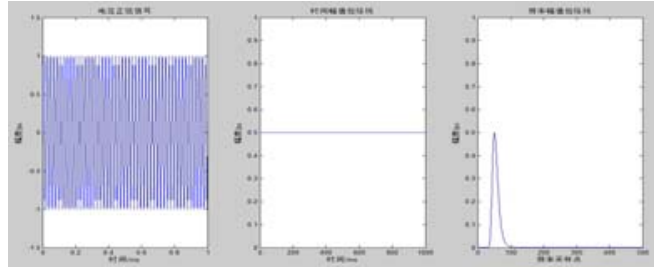

(a) Characteristic curve of the ideal voltage signal

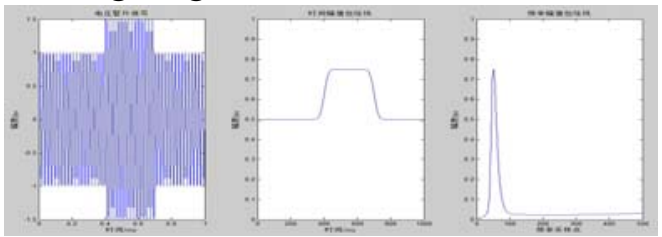

(c) Characteristic curve of the transient voltage signal

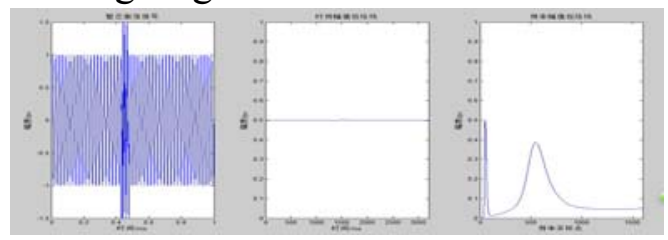

(e) Characteristic curve of the transient oscillation signal

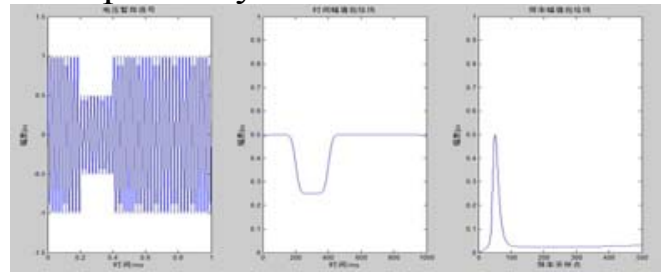

(b) Characteristic curve of the transient decline voltage signal

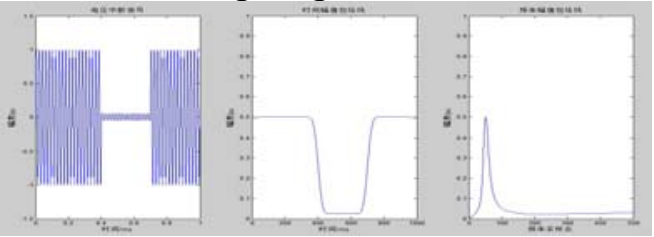

(d) Characteristic curve of the interrupt voltage signal

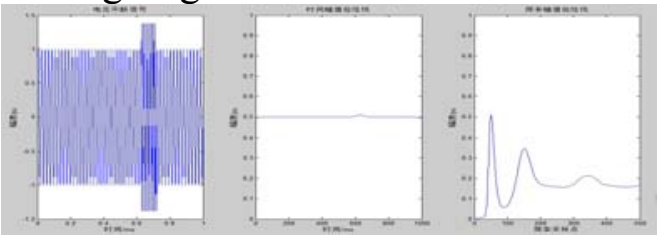

(f) Characteristic curve of the transient harmonic signal

Fig.2 Characteristic curve of the transient disturbance

From the above diagram, if can be seen that the time-frequency characteristics of every kind of disturbance signal can be shown intuitively by S-transform.

\section{Feature extraction of disturbance signals}

Eight feature characteristics were extracted from S-transform in time-frequency matrix separately for automatic identification, according to the analysis of the characteristic curve of the disturbance signal. The eight feature characteristics: the average value of fundamental frequency amplitude F1, the sample point number of amplitude greater than standard values' $105 \%$ in fundamental frequency amplitude F2 (standard amplitude $\mathrm{M}=0.4959)$, the sample point number of amplitude less than standard values' $95 \%$ in fundamental frequency amplitude F3, the sample point number of amplitude less than standard values' $10 \%$ in fundamental frequency amplitude F4, wave number of frequency envelope F5, mean value great than or equal to fundamental frequency extracted from time envelope of row vector maximums F6, standard deviation of time-domain envelope F7, standard deviation of frequency spectrum F8. Table 1 shows one feature vector of the six kinds of disturbance signals. 
Table 1. The feature vector of six kinds of disturbance signals

\begin{tabular}{ccccccccc}
\hline type of & \multicolumn{7}{c}{ characteristic quantity } \\
\cline { 2 - 10 } disturbance & F1 & F2 & F3 & F4 & F5 & F6 & F7 & F8 \\
\hline S1 & 0.4959 & 0 & 0 & 0 & 1 & 0.5 & $6.21 \mathrm{E}-16$ & 0.0829 \\
S2 & 0.4875 & 0 & 124 & 0 & 1 & 0.4905 & 0.0199 & 0.0798 \\
S3 & 0.5072 & 106 & 0 & 0 & 1 & 0.5138 & 0.0369 & 0.1092 \\
S4 & 0.2972 & 0 & 476 & 353 & 1 & 0.2651 & 0.2283 & 0.0790 \\
S5 & 0.4958 & 0 & 0 & 0 & 2 & 0.5 & 0.00032 & 0.1013 \\
S6 & 0.4954 & 0 & 0 & 0 & 4 & 0.4990 & 0.0024 & 0.1632 \\
\hline
\end{tabular}

Qualitative analysis from the table we could get that, the ideal voltage signal, the transient oscillation signal, and the transient harmonic signal could be separated from the transient decline voltage signal, the transient rise voltage signal, and the interrupt voltage signal by characteristic F1; the transient rise voltage signal could be separated from the transient decline voltage signal and the interrupt voltage signal by characteristic F2, F3, F4, the transient harmonic signal and the transient oscillation signal could be separated from the rest disturbance signal by characteristic F5, F6, the transient harmonic signal and the transient oscillation signal could be separated from the rest disturbance signal by characteristic F7, F8.

\section{Fuzzy K-Nearest Neighbor}

KNN algorithm was presented by the Cover and Hart in 1968, it is a kind of lazy learning algorithm based on statistics. The basic thought of KNN: first calculate the Euclidean distance between the classifying sample $X$ and the training samples of the training sample set according to the distance metric function, then sorted the calculated distances, selected $\mathrm{K}$ nearest training samples to the classifying sample $\mathrm{X}$ as $\mathrm{K}$ nearest neighbor of $\mathrm{X}$. Classified samples $\mathrm{X}$ as such category if the majority of the $\mathrm{K}$ nearest neighbor belonged to the category ${ }^{[13]}$.

KNN of the unknown sample was choose by KNN algorithm, classified unknown sample as such category if the majority of the $\mathrm{K}$ nearest neighbor belonged to the category. As figure 1 shows , assume there were three sample sets and 1 unknown sample, $K=5$, from the figure we can see that three belongs to $\omega 1$, one belongs to $\omega 2$, and one belongs to $\omega 3$ of the five nearest training sample. The unknown sample was classified into the category $\omega 1$ according to the rules of KNN decision ${ }^{[14]}$.

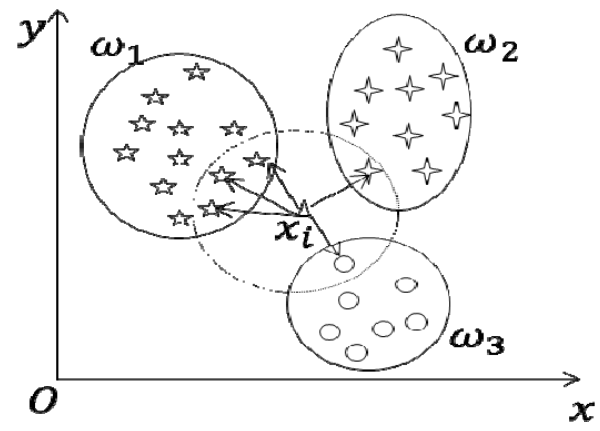

Fig.3 Schematic diagram of KNN algorithm

But it would reduce the classification accuracy of KNN if only considered the $\mathrm{K}$ sorted nearest neighbor and ignored the different distance between samples, when the distribution of the learning samples category was unevenness. Fuzzy KNN was introduced for the solving of this problem. Fuzzy KNN is a kind of supervised learning techniques which combined the fuzzy theory with the $\mathrm{k}$ nearest neighbor algorithm. It obfuscated the distance between the unknown samples and k nearest neighbors, and set the corresponding membership degree for each category, which was not as simple as KNN to categorize unknown samples as "belong to" or "does not "belong to" ${ }^{[15]}$.

\section{Simulation results and analysis}

600 training samples (each kind signal 100 samples) were produced using Matlab, 20 test 
samples was produced by each signal. Classification results are shown in table 2 .

Table 2 Result of classification

\begin{tabular}{ccccccc}
\hline type of signals & S1 & S2 & S3 & S4 & S5 & S6 \\
\hline S1 & 20 & 0 & 0 & 0 & 0 & 0 \\
S2 & 0 & 20 & 0 & 2 & 0 & 0 \\
S3 & 0 & 0 & 20 & 0 & 0 & 0 \\
S4 & 0 & 0 & 0 & 18 & 0 & 0 \\
S5 & 0 & 0 & 0 & 0 & 20 & 0 \\
S6 & 0 & 0 & 0 & 0 & 0 & 20
\end{tabular}

As the $6 * 6$ mixed matrix showed in Table 2 , the diagonal element represented the number of correct classification and the off-diagonal element represented the number of error classification. Among which the accuracy of each classification was the ratio of the correct number and the number of test samples. Table 3 shows the classification accuracy and the overall classification accuracy of each signal. The simulation result diagram is shown in figure 4 .

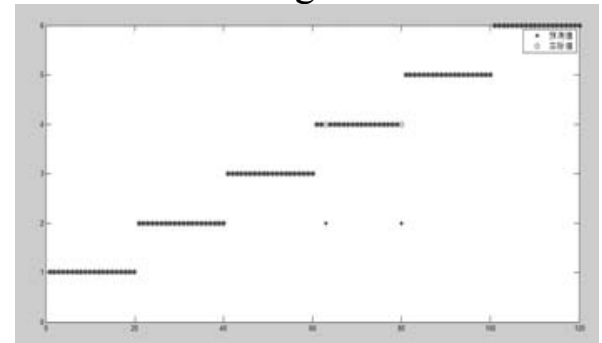

Fig.4 Diagram of simulation testing

Table 3 analysis of the classification results

\begin{tabular}{lccc}
\hline type of signals & $\begin{array}{c}\text { number of } \\
\text { test samples }\end{array}$ & $\begin{array}{c}\text { number } \\
\text { Classified } \\
\text { correctly }\end{array}$ & of accuracy \\
\hline $\begin{array}{l}\text { the ideal voltage signal } \\
\text { the transient decline voltage }\end{array}$ & 20 & 20 & $100 \%$ \\
$\begin{array}{l}\text { signal } \\
\text { the transient rise voltage signal }\end{array}$ & 20 & 20 & $100 \%$ \\
the interrupt voltage signal & 20 & 20 & $100 \%$ \\
the transient oscillation signal & 20 & 18 & $90 \%$ \\
the transient harmonic signal & 20 & 20 & $100 \%$ \\
the overall accuracy & & 20 & $100 \%$ \\
\hline
\end{tabular}

From the analysis of the simulation results, six kinds of signals can be identified and classification effectively through the proposed method. The recognition rate of each disturbance signal was high.

\section{Conclusion}

This paper processed six kinds of typical disturbance signals by S-transform, got corresponding mode time-frequency matrix and extracted eight effective characteristics. Classified six kinds of signals combined with the FKNN classifier. As a kind of supervised learning algorithm, FKNN processed each unknown sample with the same algorithm, and there is no convergence phenomenon for the un-iterative process of this algorithm. Valued the fuzzy weighted adjustment factor $\mathrm{m}=2$, and tested the influence of different $m$ values on FKNN classification accuracy within the scope of $[1,3]$ by step value of 0.1 , the results showed that there were no significant differences. The overall recognition rate of six kind disturbance signals was $98.3 \%$ when set $\mathrm{m}=2, \mathrm{k}=3$, the general classification effect was fine. 


\section{Acknowledgement}

This study was supported by QingHai Province Key Laboratory of Photovoltaic grid connected power generation technology (Grant No. SGQHDKYODWJS1500069)

\section{References}

[1] Juan Fu, Hanyong Zhou, Qin Jiang. Power quality detection and simulation using S-transform [J]. Power System Protection and Control. 200937 (21) 86-90.

[2] Bhends C N, Mishra S, Panigrahi B K. Detection and classification of power quality disturbances using S-transform and modular neural network[J]. IEEE Transactions on Power Delivery, 2008 23(1) 122-128.

[3] Xinhua Li. The Study of transient power quality problems based on S-transform and RBF neural network[J]. Automation of Electric Power System. 2011.

[4] Kaewarsa, S. Classification of power quality disturbances using S-transform based artificial neural networks[J]. Intelligent Computing and Intelligent Systems(ICIS). $20091566-570$.

[5] Na Zhang. Study on transient power quality based on S-transform[D]. Xi'an University Of Science And Technology, 2008.

[6] Rui Zhang, Tao Zhang, Hongjiao Yao, et al.. The classification and recognition of power quality disturbance based on S-transform[J]. Measurement, Information and Control (MIC). 20122663 667.

[7] Naik, C.A, Kundu, P. Identification of short duration power quality disturbances employing S-transform[J]. Power and Energy Systems (ICPS), 2011 1-5.

[8] Ganyun Lv, Haozhong Cheng, Jinju Zheng, et al. Power Quality Disturbances Detection and Identification Based on S-Transform and Multi-Lay SVMs[J]. Transactions of China Electrotechnical Society. 200606 (01) 121-125.

[9] Khoa Ngominh, Viet Dinhthanh, Hieu Nguyenhuu. Classification of Power Quality Disturbances Using Wavelet Transform and K-Nearest Neighbor Classifier[J]. Industrial Electronics(ISIE). 2013 1-4.

[10] Dash P K, Panigrahi K B, Panda G. Power Quality Analysis Using S-Transform[J]. Power Delivery, 2003 18(2) 406-411.

[11] Fengzhan Zhao, Rengang Yang. Power Quality Disturbances Classification Based on S-Transform and Time Domain Analysis[J]. Power System Technology. 2006 30(15) 90-94.

[12] Xin Liu, Honggeng Yang, Jun Zhao. Classification of short power quality disturbances based on local similarity of the time-frequency matrix of S-transform mode[J]. Automation of Electric Power Systems. 2009 33(8) 61-65.

[13] He Q P, Jin Wang. Fault detection using the K-Nearest neighbor rule for semiconductor manufacturing processes [J].Semiconductor Manufacturing. 2007 20(4) 345-354.

[14] Fan Liu, Yun Zhang, Xiao Yaol. Recognition of PD mode based on KNN algorithm for converter transformer[J]. Electric Power Automation Equipment. 2013 33(5) 89-93.

[15] Wenqian Shang, Youli Qu, Houkuan Huang. An Adaptive Fuzzy KNN Text Classifier Based on Gini Index Weight[C]. IEEE Computer Society, 2006 24(4) 87-90. 